\title{
A GNSS Payload for CubeSat Precise Orbit Determination
}

\section{Other Conference Item}

Author(s):

Chen, Kangkang; Rothacher, Markus; Müller, Lukas; Kreiliger, Flavio; De Florio, Sergio

Publication date:

2020-04-20

Permanent link:

https://doi.org/10.3929/ethz-b-000459705

Rights / license:

Creative Commons Attribution 4.0 International

Originally published in:

EGUsphere 
EGU2020-10519

https://doi.org/10.5194/egusphere-egu2020-10519

EGU General Assembly 2020

(c) Author(s) 2020. This work is distributed under

the Creative Commons Attribution 4.0 License.

\title{
A GNSS Payload for CubeSat Precise Orbit Determination
}

\author{
Kangkang Chen ${ }^{1}$, Markus Rothacher ${ }^{1}$, Lukas Müller ${ }^{1}$, Flavio Kreiliger ${ }^{2}$, and Sergio De Florio ${ }^{3}$ \\ ${ }^{1}$ ETH Zurich, Institute of Geodesy and Photogrammetry, D-BAUG, Zürich, Switzerland (kachen@ethz.ch) \\ ${ }^{2}$ Lucerne University of Applied Sciences and Arts, Switzerland \\ ${ }^{3}$ Astrocast SA, 1024 Ecublens, Switzerland
}

Global Navigation Satellite Systems (GNSS) have been used as a key technology for satellite orbit determination for about 30 years. With the increasing popularity of miniaturized satellites (e.g., CubeSats that are nanosatellites based on standardized $10 \mathrm{~cm}$-sized units) the need for an adapted payload for orbit determination arises. We developed a small-size versatile GNSS payload board using commercial off-the-shelf single-frequency GNSS receivers with extremely small weight $(1.6 \mathrm{~g})$, size $\left(12.2 \times 16.0 \times 2.4 \mathrm{~mm}^{3}\right)$ and power consumption $(100 \mathrm{~mW})$. The board features two separate antenna connectors and four GNSS receivers - two connected to each antenna. This redundancy lowers the risk of a total payload failure in case one receiver is malfunctioning.

Two prototypes of the GNSS positioning board have been successfully launched onboard the Astrocast-01 and -02 3-unit cube satellites with altitudes of 575 and $505 \mathrm{~km}$, respectively. The multiGNSS receivers are capable of tracking the GNSS satellites of the four major systems, i.e., GPS, GLONASS, BeiDou and Galileo. In addition, both satellites are equipped with a small array of three laser retroreflectors enabling orbit validation with Satellite Laser Ranging (SLR). After the two precursor missions, a constellation of 80 satellites is planned, allowing the formation and computation of a highly uniform polyhedron in space with cm-accuracy, relevant for geocenter, reference frame, and GNSS orbit determination.

At present, we have continuous receiver PVT solutions available. The real-time onboard orbit determination results indicate that the receivers perform very well on both satellites. The RMS of a daily orbit fitting is, after removing one or the other outlier, at the level of 2-5 meters despite errors caused by the ionosphere and the orbit model. For a few satellite arcs, the recording of GNSS raw phase and code data was enabled, allowing orbit determination in a post-processing mode. This allows a better assessment of the achievable orbit quality and an overall performance estimation. The tests performed so far include the improvement of the orbit quality by eliminating the ionospheric refraction based on a linear combination of phase and code observations, the comparison of various single-system solutions and advances in combining the different tracking systems for orbit determination. In collaboration with the Zimmerwald Observatory in Switzerland a first SLR campaign was conducted that successfully tracked both nanosatellites. The SLR measurements with their high accuracy were then analyzed to validate the orbits of the Astrocast satellites derived from GNSS measurements. 
We will present details on the payload board, on the results of the orbit determination in real-time and in post-processing mode based on the low-cost single-frequency multi-GNSS receivers onboard the satellites and on the SLR orbit validation.

Keywords: CubeSat; GNSS payload; LEO orbit determination; low-cost; ionospheric refraction; linear combination; SLR 